\title{
PERFIL DOS TRABALHADORES SUBMETIDOS À AUDIOMETRIA ATENDIDOS PELO SERVIÇO SOCIAL DA INDÚSTRIA DA UNIDADE SUDOESTE - BAHIA
}

\author{
Jéssica Santos Tiago \\ Graduanda do curso de Enfermagem Independente \\ do Nordeste. \\ Giovana Fernandes Araújo \\ Enfermeira, Mestre em Meio Ambiente e \\ Sustentabilidade. Especialista em Enfermagem do \\ Trabalho e Saúde da Família. Professora do curso \\ de Enfermagem da Faculdade Independente do \\ Nordeste.
}

Endereço para correspondência: jeltiago@ hotmail.com

\begin{abstract}
Resumo
No âmbito da Saúde do Trabalhador, a enfermagem tem papel importante na prevenção das doenças ocupacionais. A Perda Auditiva Induzida por Ruído (PAIR) tornou-se um problema de saúde pública acarretando danos à saúde do trabalhador. O objetivo deste estudo foi identificar o perfil dos trabalhadores atendidos pelo Serviço Social da Indústria da Unidade Sudoeste - Bahia submetidos à audiometria. Trata-se de um estudo descritivo de natureza quantitativa. A pesquisa foi realizada por meio da análise de 100 prontuários dos colaboradores do ramo ceramista submetidos à consulta clínica ocupacional e realização do exame audiométrico, atendidos numa empresa de Serviço Social de Indústria, especializado na área de Segurança e Saúde do Trabalhador. Os resultados mostraram que das audiometrias avaliadas, $49 \%$ tiveram algum tipo de alteração. Pode-se relacionar também a função ajudante de produção com a alteração da audiometria $(p=0,0027)$ e o tempo de função maior de 5 anos e alteração da audiometria $(p=0,000)$. A PAIR neste estudo foi inferior em comparação com a prevalência nos demais artigos revisados. Com base nos resultados encontrados, emerge a necessidade de aprimoramento de programas de conservação auditiva (PCA) nas indústrias. Contudo o presente estudo sugere a necessidade de aperfeiçoamento de práticas de prevenção no campo da Enfermagem do Trabalho, com foco em ações voltadas para a informação do trabalhador exposto ao ruído ocupacional.
\end{abstract}

Palavras-chave: Perda auditiva; Saúde do trabalhador; Enfermeiro.

\section{PROFILE OF EMPLOYEES UNDERGOING AUDIOMETRY ATTENDED BY SOCIAL SERVICES INDUSTRY DRIVE SOUTHWEST - BAHIA}

\begin{abstract}
Under the Occupational Health, nursing has an important role in the prevention of occupational diseases. The Noise Induced Hearing Loss (NIHL) has become a public health problem causing damage to workers' health The objective is to identify the profile of workers attended the Service Industry Drive Southwest - Bahia underwent audiometry. This is a descriptive study of a quantitative nature. The survey was conducted through the analysis of 100 medical records of employees of the branch potter underwent clinical consulting and occupational Audiometric testing, served in a company of Social Service for Industry, specialized in the field of Safety and Occupational Health. The results showed that the Audiometries, $49 \%$ had some type of change. One can also relate the function helper production with altered auditory $(\mathrm{p}=0.0027)$ and time function over 5 years and altered audiometry $(\mathrm{p}=0.000)$. The NIHL in this study was lower compared to the prevalence in other articles reviewed. Based on these results, emerges the need of improvement of hearing conservation programs (HCP) industries. However this study suggests the need for improvement of prevention practices in the field of Occupational Health Nursing, with a focus on actions aimed at information workers exposed to occupational noise.
\end{abstract}

Descriptors: Hearing loss; Health worker; Nurse. 


\title{
PERFIL DE LOS TRABAJADORES QUE EXPERIMENTARON LA AUDIOMETRÍA ATENDIDA POR SERVIÇO SOCIAL DA INDÚSTRIA SUDOESTE UNIDAD - BAHIA
}

\begin{abstract}
Resumen
En la salud del trabajador, la enfermería tiene papel importante en la prevención de las enfermedades profesionales. Pérdida de audición inducida por el ruido (nihl) se ha convertido en un problema de salud pública que causan daño a la salud del trabajador. El objetivo de este estudio fue identificar el perfil de los trabajadores atendidos por unidad de Serviço Social da Indústria-Bahia suroeste sometida a la audiometría. Este es un estudio descriptivo de carácter cuantitativo. La encuesta fue realizada a través del análisis de 100 cartas de empleados de la ceramista sometidos a Clinica ocupacional consulta y examen audiométrico, servido en una empresa de Servicio Social de la industria, especializada en el área de salud y seguridad de los trabajadores. Los resultados mostraron que de audiometrias evaluados, $49 \%$ tenía algún tipo de cambio. También puede conectar la función de ayudante de producción cambiando la audiometría $(\mathrm{p}=0,0027)$ y función mayor de 5 años y modificación de audiometría $(\mathrm{p}=0,000)$. El par en este estudio fue menor comparado con la prevalencia en otros artículos revisados por pares. Basado en los resultados encontrados, surge la necesidad de mejora de los programas de conservación (PCA) en las industrias de la audición. Sin embargo, este estudio sugiere la necesidad de mejorar las prácticas de prevención en el campo de la enfermería, con énfasis en acciones dirigidas a informar a los trabajadores expuestos al ruido ocupacional.
\end{abstract}

Palabras clave: Pérdida de la audición; La salud del trabajador; Enfermera.

\section{INTRODUÇÃO}

As doenças ocupacionais tornaram-se objeto de estudo crescente por trazer prejuízos relevantes à saúde do trabalhador, constituindo assim um importante problema de saúde pública. Dentre as principais doenças ocupacionais está a Perda Auditiva Induzida por Ruído (PAIR). A perda auditiva quando relacionada ao ruído ocupacional é caracterizada por uma diminuição gradativa da acuidade auditiva devido à exposição contínua a níveis de ruído acima do permitido. ${ }^{(1)}$

O ruído é o principal agente físico presente em diferentes categorias profissionais. A exposição a níveis de pressão sonora elevada em função de sua duração, frequência, intensidade e suscetibilidade individual, além de acarretar múltiplas consequências ao organismo humano, constitui um dos principais problemas de audição, podendo causar perda auditiva. $^{(2)}$

Os principais sintomas da PAIR definidos pelo Ministério da Saúde são: a perda auditiva; zumbido; padrão de sono irregular; tontura, cefaléia, irritabilidade e dificuldade na compreensão da fala. Ainda como consequências do ruído excessivo podem surgir também dificuldades em ouvir sons domésticos e problemas de compreensão das falas em locais amplos (salões, igrejas), e consequente constrangimento e isolamento social devido uma autoimagem que o caracteriza como velho, surdo ou incapaz. ${ }^{(3)}$ 
Segundo Guida $^{(4)}$ a perda de audição além de provocar irritabilidade e atrapalhar a comunicação ela pode acarretar o desenvolvimento de patologias como a hipertensão arterial e insônia, o que interfere na qualidade de vida do trabalhador e no surgimento de distúrbios psicossociais.

A PAIR é o agravo mais frequente à saúde do trabalhador e se encontra presente nos diversos ramos de atividade, dentre eles: siderurgia, metalurgia, gráfica, vidraria, construção civil, entre outros. ${ }^{(5)}$

A depender do tempo de exposição e da descoberta da doença, esta se torna irreversível e compromete a qualidade de vida e, diante desta problemática, as intervenções que se deve ter no ambiente de trabalho são indispensáveis para preservar a saúde do trabalhador. A prevenção contra a PAIR depende do trabalho desenvolvido pela equipe do serviço de Saúde do Trabalhador e da fiscalização das empresas, no que diz respeito aos ruídos que ultrapassam os limites de tolerância, e do entendimento por parte dos trabalhadores quanto ao uso contínuo do protetor auricular em zonas de ruído elevado. ${ }^{(4)}$

O uso de Equipamentos de Proteção Individual (EPI) auditivos para atenuar o ruído ocupacional é amplamente difundido. Os administradores frequentemente têm a compreensão errônea de que o controle do ruído através de medidas de engenharia é de difícil execução e dispendioso e com isso elas são proteladas, em alguns casos. Muitas vezes, toda a prevenção da perda auditiva induzida pelo ruído ocupacional está restrita ao EPI auditivo, dada a crença de que a simples utilização do equipamento é a medida principal e plenamente satisfatória como procedimento de conservação auditiva. ${ }^{(6)}$

O Ministério do Trabalho, por meio da portaria 3.214, aprovou as NR's, a partir de 1978, as quais estabelecem obrigações quanto ao EPI, exames médicos, e quanto aos níveis de tolerância de ruído, de acordo com a NR6, NR7 e NR15, respectivamente. ${ }^{(7)}$

As empresas devem manter de acordo com as NR's do Ministério do Trabalho e Emprego (MTE), um Programa de Prevenção de Riscos Ambientais (PPRA - na portaria/MTb no 19/1998, NR9), que considera os diversos riscos que podem existir no trabalho, que devem ser quantificados e identificados, assim a partir dessas informações é possível direcionar ações do Programa de Controle Médico de Saúde Ocupacional (PCMSO NR7) que irá proceder para as avaliações de saúde dos trabalhadores. ${ }^{(3)}$

Os fatores de risco para PAIR e trauma acústico podem ser classificados em fatores de risco ambientais dentre estes existe o ruído que provoca danos auditivos, conforme as Normas 
Regulamentadoras (NR) 15 da portaria/MTb, no 3.214/1978, nos anexos 1 e 2 estabelece os limites de tolerância para ruído contínuo ou intermitente em máxima exposição diária permissível de 8 horas com o nível de ruído equivalente a $85 \mathrm{~dB} .^{(8)}$

A prevalência da PAIR no meio de trabalho ainda é alta e esta perda de audição pode prejudicar a qualidade de vida afetando as relações sociais, de comunicação e de trabalho, evidencia-se a importância de ações preventivas e coletivas que visem à conservação auditiva e da saúde em geral, preservando assim a qualidade de vida destes. ${ }^{(9)}$

Prevenção é entendida como a adoção de intervenções que têm objetivo a eliminação e/ou controle do ruído. Assim existe um "protocolo" que apresenta as recomendações propostas pela Occupational Safaty \& Health Administration (OSHA) em 1983 e pelo National Institute for Occupational Safety and Health (NIOSH), que tem como base os aspectos do Programa de Conservação Auditiva (PCA): a avaliação e monitoramento da exposição; as medidas de controle ambiental; a avaliação e monitoramento audiológico; o uso de protetores auriculares; os aspectos educativos; e, a avaliação de eficácia. ${ }^{(1)}$

O PCA tem como objetivo a identificação de riscos, monitoramento auditivo, medida de proteção contra ruído e medidas educativas. Um Programa Preventivo voltado ao trabalhador exposto ao ruído ocupacional deve ter três dimensões à análise do ambiente de trabalho, a realização de ações educativas e estudos do perfil epidemiológico avaliando as condições auditivas individuais e coletivas. ${ }^{(10)}$

A conscientização é um componente importante em qualquer Programa de Conservação Auditiva (PCA), assim é de suma importância à conscientização do trabalhador diante o uso do protetor auricular que deve ser de conforto ao usuário, pois a supervisão e o treinamento serão efetivos se houver a participação de todos. ${ }^{(11)}$

Observa-se que a presença do Enfermeiro do Trabalho, como integrante da equipe do Serviço de Saúde do Trabalhador, contribui substancialmente para a promoção da saúde e a percepção que os trabalhadores têm sobre a perda auditiva. A enfermagem deve centrar-se na programação, organização e supervisão do trabalho e no comportamento dos trabalhadores no sentido de produzir uma vida mais saudável. ${ }^{(6)}$

Segundo Brasileiro ${ }^{(12)}$ a enfermagem também deve estar atenta à inspeção periódica dos aparelhos, o uso de aparelhos ruidosos somente o tempo necessário e a realização de testes audiométricos regularmente para que desse modo ocorra a diminuição da exposição ao 
ruído e monitorizar seus efeitos. Esta atuação conjunta da equipe acarreta num diagnóstico precoce da doença e no desenvolvimento de soluções para a saúde preventiva e curativa.

A proposição deste estudo é justificada, em primeiro lugar, tendo em vista que esta pesquisa contribuirá para o desempenho da Saúde Ocupacional, uma vez que, dando ênfase à saúde do trabalhador as empresas proporcionarão um ambiente mais seguro, jornadas de trabalho sem prejuízos a saúde, responsabilidade diante do colaborador e agregação de uma imagem de qualidade aos serviços prestados. Em segundo lugar, porque surgiu o interesse em realizar este estudo durante o período da realização de estágio extracurricular onde foi possível perceber alterações nos exames audiométricos de trabalhadores atendidos e acompanhados pela Segurança e Saúde do Trabalhador no SESI Unidade Sudoeste.

Nesta perspectiva objetivou-se identificar qual é o perfil dos trabalhadores atendidos pelo Serviço Social da Indústria da Unidade Sudoeste - Bahia submetidos à audiometria.

\section{METODOLOGIA}

Trata-se de um estudo descritivo e de natureza quantitativa ${ }^{(13)}$, realizada em uma empresa de Serviço Social de Indústria, especializado na área de Segurança e Saúde do Trabalhador. É uma instituição privada, sem fins lucrativos, integrante da Federação das Indústrias do Estado da Bahia (FIEB), cujo ramo de atendimento está voltado à indústria.

A coleta de dados foi realizada por meio da análise de 100 prontuários dos colaboradores do ramo ceramista submetidos à consulta clínica ocupacional, no período de maio de 2012 a maio de 2013. O critério de inclusão foi prontuários que constasse a realização e resultado de audiometria do trabalhador.

Foi utilizado como instrumento de pesquisa um roteiro composto pelas variáveis: sexo, idade, nível de escolaridade, empresa, setor, função operante, tempo de função e resultados dos exames audiométricos no aspecto de ter ocorrido alteração ou não na audição do trabalhador.

Os dados foram inicialmente inseridos em uma planilha do Microsoft Excel 2010, sendo em seguida transportados para uma planilha do programa estatístico SPSS versão 20.0, no qual foi realizada uma análise estatística descritiva e aplicação do teste Qui-quadrado. 
A pesquisa foi autorizada pelo Comitê de Ética em Pesquisa (CEP) da instituição proponente, e está de acordo com a Resolução 196/96 do Conselho Nacional de Saúde, sendo aprovada pelo protocolo $\mathrm{n}^{\mathrm{o}}$ 17597913.5.0000.5578.

\section{RESULTADOS E DISCUSSÕES}

A PAIR é considerada uma das doenças ocupacionais mais prevalentes em todo o mundo. ${ }^{(14)}$ As contínuas mudanças que se processam no mundo do trabalho e as distintas características regionais do Brasil têm múltiplos efeitos sobre a saúde e configuram perfis epidemiológicos mutantes na população trabalhadora. Qualquer tentativa de estudar os agravos à saúde do trabalhador no país é sempre parcial, pois não há uma abordagem única que permita uma visão acurada do sistema. ${ }^{(15)}$

Os dados epidemiológicos no Brasil são escassos, não há registros que caracterizem a real situação, e, portanto, os dados disponíveis dão ideia parcial do risco relacionada à perda auditiva. $^{(8)}$

A tabela 1 a seguir apresenta o perfil sociocultural dos trabalhadores do ramo ceramista atendidos no Serviço Social da Indústria da Unidade Sudoeste - Bahia, constatando que no presente estudo $90,0 \%$ dos indivíduos eram do sexo masculino, com faixa etária prevalente de 26 a 35 anos $(34,0 \%)$ e nível de escolaridade referente ao fundamental incompleto $(27,0 \%)$. 
Tabela 1 - Perfil sociocultural dos trabalhadores do ramo ceramista atendidos no Serviço Social da Indústria da Unidade Sudoeste- Bahia. Vitória da Conquista/BA, 2013.

\begin{tabular}{lcc}
\hline Variáveis de Estudo & $\mathbf{n}$ & $\mathbf{\%}$ \\
\hline Sexo & & \\
Masculino & 90 & 90,0 \\
Feminino & 10 & 10,0 \\
Nível de Escolaridade & & \\
Não alfabetizado & 7 & 7,0 \\
Ensino primário completo & 6 & 6,0 \\
Ensino primário incompleto & 6 & 6,0 \\
Ensino fundamental completo & 25 & 25,0 \\
Ensino fundamental incompleto & 27 & 27,0 \\
Ensino médio completo & 21 & 21,0 \\
Ensino médio incompleto & 7 & 7,0 \\
Ensino superior incompleto & 1 & 1,0 \\
Faixa Etária & & \\
15 a 25 anos & 14 & 14,0 \\
26 a 35 anos & 34 & 34,0 \\
36 a 45 anos & 30 & 30,0 \\
46 a 55 anos & 15 & 15,0 \\
Acima de 55 anos & 7 & 7,0 \\
\hline Total & $\mathbf{1 0 0}$ & $\mathbf{1 0 0 , 0}$ \\
\hline
\end{tabular}

Fonte: Elaborado pelas autoras baseado em dados coletados

Conforme dados da Tabela 1 houve prevalência significativa do gênero masculino sobre o feminino representando 90,0\% dos trabalhadores. Segundo Miranda; Dias; Pena; Nobre; Aquino ${ }^{(16)}$ após a realização de uma pesquisa sobre Perda Auditiva Induzida pelo Ruído em trabalhadores industriais da região Metropolitana de Salvador avaliou 7.925 trabalhadores de 44 empresas industrias em atividade, observando a prevalência do gênero masculino.

É possível que esta característica do gênero masculino dos trabalhadores que participaram do estudo esteja relacionada à exigência de esforço físico que o ramo ceramista necessita. Como o serviço de modo geral é pesado, os homens são maioria.

O presente estudo corrobora o de Maciel; Valle; Maciel ${ }^{(17)}$ sobre Homens do barro e estratégias empresariais: uma análise da relação capital-trabalho no polo oleiro-cerâmico de Iranduba-AM o qual verificou que dos 50 trabalhadores entrevistados, os homens se constituem a maioria, sendo que apenas cinco mulheres fazem parte do quadro de trabalhadores. 
Ratifica o estudo de Cunha; Costa; Lacomblez ${ }^{(18)}$ sobre idade e saúde na organização do trabalho: definir novas estratégias de prevenção dos riscos no setor de cerâmica que constatou que dos 155 sujeitos da pesquisa, 91 (58,7\%) eram do sexo masculino.

Nota-se que o nível de escolaridade que prevaleceu foi o ensino fundamental incompleto $(27 \%)$ comprovando assim a pesquisa de Fuini ${ }^{(19)}$ sobre um estudo de mercado de trabalho em arranjo produtivo local (APL): Território e produção cerâmica em Santa Gertrudes/SP que apresentou uma instrução média de mão-de-obra a partir dos módulos completos e incompletos cursados pelos empregados, predomínio de instrução até o nível fundamental ou (8a série).

Maciel; Valle; Maciel ${ }^{(17)}$ no estudo sobre Homens do barro e estratégias empresariais: uma análise da relação capital-trabalho no polo oleiro-cerâmico de Iranduba-AM verificaram a grande porcentagem de trabalhadores que estudaram até o Ensino Fundamental, cerca de $44 \%$.

No que diz respeito aos trabalhadores do ramo ceramista é possível relacionar o baixo nível de escolaridade com o fato de que as atividades exercidas por eles não necessitam de qualificação profissional.

Foi observado também que a população estudada abrange trabalhadores na faixa etária de 15 a mais de 55 anos. Nota-se que esta é uma população de adultos jovens prevalecendo idades compreendidas entre 26 e 35 anos.

Convalida o estudo de Ferreira, Peloggia, Pirinoto e Bernardi ${ }^{(20)}$ sobre influência da conservação do protetor auricular na prevenção da perda auditiva por níveis de pressão sonora elevados o qual verificou a prevalência de profissionais adultos jovens que estavam na faixa etária de 25 a 40 anos, sendo que as idades dos entrevistados variaram de 24 a 54 anos.

O presente estudo revalida o de Isidoro e Moraes ${ }^{(21)}$ que encontraram maior número de trabalhadores na faixa etária de 28,4 anos $( \pm 8,54)$ na pesquisa sobre "Análise das atividades do setor de classificação de uma indústria de revestimentos cerâmicos".

Igualmente jovem foi à população de trabalhadores que Maciel; Valle; Maciel ${ }^{(17)}$ encontrou em seu estudo sobre uma análise da relação capital-trabalho no polo oleirocerâmico de Iranduba-AM na qual 50 trabalhadores estavam na faixa etária de 25 a 34 anos como nos dados encontrados na presente pesquisa. 
A tabela 2 a seguir apresenta à caracterização sobre o trabalho dos colaboradores do ramo ceramista. Verificou-se uma maior distribuição de trabalhadores do setor de produção e trabalhadores que exercem suas funções a mais de 5 anos nesta empresa $(35,0 \%)$.

Tabela 2 - Caracterização sobre o Trabalho dos colaboradores do ramo ceramista atendidos no Serviço Social da Indústria da Unidade Sudoeste- Bahia. Vitória da Conquista/BA, 2013

\begin{tabular}{lcc}
\hline Variáveis de Estudo & $\mathbf{n}$ & \% \\
\hline Setor de Trabalho & 23 & 23,0 \\
Administrativo & 6 & 6,0 \\
Desenforna & 7 & 7,0 \\
Enforna & 8 & 8,0 \\
Expedição & 17 & 17,0 \\
Manutenção & 32 & 32,0 \\
Produção & 7 & 7,0 \\
Queima & & \\
Tempo de Função & 23 & 23,0 \\
0 a 1 ano & 22 & 22,0 \\
1 a 3 anos & 20 & 20,0 \\
3 a 5 anos & 35 & 35,0 \\
Acima de 5 anos & 100 & 100,0 \\
\hline Total &
\end{tabular}

Fonte: Elaborado pelas autoras baseado em dados coletados

Conforme dados da Tabela 2 houve prevalência do setor produção, representando $32 \%$ dos trabalhadores. Segundo Loyola ${ }^{(22)}$ ao realizar o estudo sobre o Programa de Capacitação de Recursos Humanos na Indústria Cerâmica do estado do Paraná notou que a função de Auxiliar Geral do setor Produção representa 51,16\% do total da mão-de-obra declarada.

Conforme é observado na Tabela 2 verificou-se que a maioria dos profissionais (35\%) possui tempo de serviço maior do que 5 anos. Este tempo de serviço acima de 5 anos pode indicar, principalmente entre profissionais de nível fundamental incompleto, a dificuldade de fixação em outro ramo ocupacional que exija maior conhecimento e que não utilize a força braçal.

A tabela 3 a seguir apresenta a alteração da audiometria dos trabalhadores do ramo ceramista atendidos no Serviço Social da Indústria da Unidade Sudoeste - Bahia, constando que $51,0 \%$ dos indivíduos não possuem comprometimento auditivo. 
Tabela 3 - Alteração dos Exames Audiométricos dos colaboradores do ramo ceramista atendidos no Serviço Social da Indústria da Unidade Sudoeste- Bahia. Vitória da Conquista/BA, 2013

\begin{tabular}{lcc}
\hline Variáveis de Estudo & $\mathbf{n}$ & \% \\
\hline Alteração da Audiometria & & \\
Sim & 49 & 49,0 \\
Não & 51 & 51,0 \\
\hline Total & 100 & 100,0 \\
\hline
\end{tabular}

Fonte: Elaborado pelas autoras baseado em dados coletados

Comparando os resultados obtidos com os dados da literatura, os achados foram superiores aos encontrados em 2002 por Araújo, ${ }^{(23)}$ que avaliou 187 audiometrias ocupacionais de trabalhadores de uma indústria metalúrgica de Goiânia, e observou que 41 (21\%) dos exames audiométricos foram sugestivos de PAIR. Em outro estudo em 2006

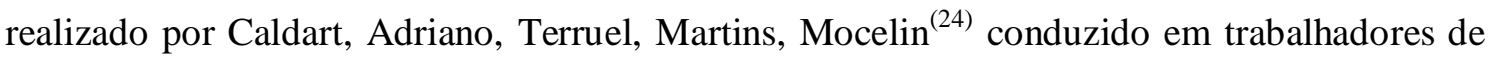
indústria têxtil, a prevalência de PAIR foi superior a obtida neste estudo, totalizando 105 trabalhadores, dentre os quais $52(28,3 \%)$ apresentavam curvas audiométricas compatíveis com PAIR.

Com a aplicação do teste do Qui-quadrado verificou-se diferença estatística entre a função ajudante de produção e alteração da audiometria $(p=0,0027)$ e entre tempo de função mais de 5 anos e alteração da audiometria $(p=0,000)$.

Esta pesquisa mostrou a relação da perda auditiva diretamente relacionada com a função ajudante de produção, referenciando assim a pesquisa de Miranda, Dias, Pena, Nobre, Aquino $^{(16)}$ sobre Perda auditiva induzida pelo ruído em trabalhadores industriais da região metropolitana de Salvador, Bahia que comprovou um aspecto importante do desgaste à saúde detectado no que diz respeito à distribuição das perdas auditivas entre as funções. As prevalências de perdas auditivas em trabalhadores em manutenção e em serviços de apoio à produção indicam que há uma população de trabalhadores expostos diretamente ao ruído ligados à produção, inclusive com maior ocorrência de PAIR.

Em relação ao tempo de função maior que 5 anos correlacionado com a perda auditiva obtida neste estudo, pode-se reafirmar com o estudo de Caldart, Adriano, Terruel, Martins, Mocelin ${ }^{(24)}$ sobre Prevalência da Perda Auditiva Induzida pelo Ruído em Trabalhadores de Indústria Têxtil demonstrou que os casos de PAIR cresceram à medida que aumentou o tempo de trabalho na indústria, e que os trabalhadores com mais de 20 anos de empresa, ou seja, de 
exposição, foram os mais afetados, com índice de $42,9 \%$. Isto reforça a afirmativa de que os efeitos auditivos dependem principalmente da intensidade e da duração da exposição ao ruído, como também demonstraram Costa ${ }^{(25)}$ e Belachew e Berhane ${ }^{(26)}$

\section{CONCLUSÕES}

Os objetivos desta pesquisa foram alcançados conforme a apresentação dos resultados. Verificou-se que quanto maior o tempo de função ou a função operante maior é a chance de desenvolver algum comprometimento auditivo. Os achados indicados contribuíram para fortalecer a necessidade de uma atenção especial para a população estudada. Todos os resultados apresentados nesta pesquisa houve em parte a influência do nível de escolaridade, tendo em vista que quanto menor o grau de escolaridade, maior é a dificuldade em compreender os riscos existentes no ambiente de trabalho e aderir o uso correto dos EPI's.

Contudo o presente estudo reafirma a importância das práticas de prevenção no campo de Enfermagem do Trabalho, com foco em ações voltadas para a informação do trabalhador exposto ao ruído ocupacional. Deste modo salienta-se a participação do Enfermeiro do Trabalho na realização de um PCA com êxito, ou seja, o desenvolvimento de medidas educativas que interfira diretamente na conscientização dos trabalhadores expostos.

\section{REFERÊNCIAS}

1. Matos MP, et al. Ruído risco e prevenção. 3ºd. São Paulo: Ed Hucitec; 1999.

2. Gonçalves CG. Saúde do trabalhador: da estruturação à avaliação de programas de preservação auditiva. São Paulo: Roca; 2009.

3 Gonçalves CG. Ministério do Trabalho. Portaria no 3.214, de 08 de junho de 1978- NR 04, NR 05, NR 06, NR 09, NR 13. Diário Oficial da República Federativa do Brasil, Brasília; 2006.

4. Guida HL. Efeitos psicossociais da perda auditiva induzida pelo ruído em ex-funcionários da indústria. ACTA ORL/Técnicas em otorrinolaringologia. 2008 [acesso em 2012 Mar 3];25(1):78-83. Disponível em: http://www.actaorl.com.br/PDF/25-01-14.pdf.

5. Buriti AKL, Farias VHV, Rosa MRD. Ocorrência de perda auditiva induzida pelo ruído em carpinteiros. Revista CEFAC 2012;14(3):413-422. 
6. Cesar-Vaz MR, Soares JFS, Almeida MCV, Cardoso LS, Bonow CA. Doenças Relacionadas ao Trabalho Autorreferidas por Trabalhadores Portuários Avulsos. Cienc Cuid Saude 2010 Out/Dez; 9(4):774-781

7. Pagliosa KM. Ruído - Relação entre saúde, trabalho e qualidade de vida. Porto Alegre: CEFAC; 1999 [acesso em 2012 Mar 3]. 42 p. Disponível em: http://www.cefac.br/library/teses/2ed3955f1763d2e1f6c6149eff3ed76.pdf

8. Brasil. Ministério da Saúde. Doenças Relacionadas ao Trabalho: manual de procedimentos para os serviços de saúde. Brasília, DF; 2001a. 580 p.

9. Lopes AC, Nelli MP, Lauris JRP, Amorim RB, Melo ADP. Condições de saúde auditiva no trabalho: investigação dos efeitos auditivos em trabalhadores expostos ao ruído ocupacional. Arq. Int. Otorrinolaringol. 2009;13(1):49-54.

10. Gonçalves CGO. Implantação de um programa de preservação auditiva em metalúrgica: descrição de uma estratégia. Distúrbios de Comunicação. 1999;16(1):43-51.

11. Vieira IL. Audiologia clínica: ruído e perda auditiva. Recife: CEFAC; 1999 [acesso em 2012 Mar 3]. 36p. Disponível em:

http://www.cefac.br/library/teses/1565f80d879b2ef53634d25e0a4f29f7.pdf

12. Brasileiro ME, Canedo MLS, Silva PF. Assistência de enfermagem em pacientes com lesões por esforço repetitivo. Revista Eletrônica de Enfermagem do Centro de Estudos de Enfermagem e Nutrição [serial on-line] 2013 [acesso em 2012 Mar 3];2(2) 1-15. Disponível: http://www.ceen.com.br/revistaeletronica

13. Menezes EM, Silva EL. Metodologia da pesquisa e elaboração de dissertação. 4. ed. Florianopólis: UFCS; 2005 [acesso em 2012 Mar 3]. 138 p. Disponível em: http://www.tecnologiadeprojetos.com.br/banco_objetos/\%7B7AF9C03E-C286-470C-9C07EA067CECB16D\%7D_Metodologia\%20da\%Pesquisa\%20e\%20da\%Disserta\%C3\%A7\%C3 $\%$ A3o\%20\%20UFSC\%202005.pdf.

14 Dias A, Cordeiro R, Corrente JE. Associação entre perda auditiva induzida pelo ruído e zumbidos, Cad. Saúde Pública. 2006;22(1):63-68.

15 Filho WV. Perfil Epidemiológico dos Trabalhadores. Revista Brasileira Medicina do Trabalho 2004; 2(2):103-117.

16 Miranda CR, Dias CR, Pena PGL, Nobre LCC, Aquino R. Perda auditiva induzida pelo ruído em trabalhadores industriais da região metropolitana de Salvador, Bahia. Inf. Epidemiol. Sus. 1998;7(1):87-94.

17. Maciel CF, Valle MIM, Maciel JMBM. Homens do barro e estratégias empresariais: uma análise da relação capital-trabalho no polo oleiro-cerâmico de Iranduba-AM. PLURAL, Revista do Programa de Pós-Graduação em Sociologia da USP; 2013 [acesso em 2012 Mar 3];20(1):9-30. Disponível em:

http://www.fflch.usp.br/ds/plural/edicoes/20_1/plural_v20n1_01.pdf 
18. Cunha L, Costa A, Lacomblez M. Idade e saúde na organização do trabalho: definir novas estratégias de prevenção dos riscos no setor de cerâmica. Kéramica n. ${ }^{\circ}$ 262, 2003 [acesso em 2012 Mar 3]. Disponível em: http://repositorioaberto.up.pt/bitstream/10216/63519/2/79013.PDF

19. Fuini LL. Estudo do mercado de trabalho em arranjo produtivo local. Sociedade \& Natureza, Uberlândia. 2008 [acesso em 2012 Mar 3];20(1):75-85; jun.. Disponível em: http://www.scielo.br/pdf/sn/v20n1/a05v20n1.pdf

20. Ferreira AP, Peloggia CCS, Pirinoto HF, Bernardi APA. Influência da Conservação do Protetor Auricular na Prevenção da Perda Auditiva por Níveis de Pressão Sonora Elevados. Revista CEFAC 2003;5(4).

21. Isidoro LS, Moraes LFS. Análise das atividades do setor de classificação de uma indústria de revestimentos cerâmicos: Sob Enfoque Ergonômico. [Monografia].

Tubarão: Universidade do Sul de Santa Catarina; 2006 [acesso em 2010 Out 29].

Disponível em: http://www.fisio-tb.unisul.br/Tccs/06b/luanaisidoro/luanaartigotcc.pdf

22. Loyola LC. Programa de Capacitação de Recursos Humanos na Indústria Cerâmica do estado do Paraná. Minerais do Paraná S/A - MINEROPAR. Curitiba; 1998.

23. Araújo AS. Perda auditiva induzida pelo ruído em trabalhadores de metalúrgica. Revista Brasileira de Otorrinolaringolia 2002;68(1):47-52.

24. Caldart AU, Adriano CF; Terruel I, Martins RF, Mocellin M. Prevalência da Perda Auditiva Induzida pelo Ruído em Trabalhadores de Indústria Têxtil. Arq. Int. otorrinolaringol. 2006 [acesso em 2012 Mar 3];10(3):192-6. Disponível em: http://www.internationalarchivesent.org/conteudo/pdfForl/380.pdf

25. Costa VHC. O ruído e suas interferências na saúde do trabalhador. Revista SOBRAC. 1994;13:912-26.

26 Belachew A, Berhane Y. Noise-induced hearing loss among textile workers. Ethiop J Health Dev. 1999;13:69-75. 